\title{
O Modelo Econômico da Empresa Quando Seus Produtos São Interdependentes Quanto À Demanda
}

\author{
Autor: Luiz João Corrar \\ Professor Doutor do Departamento de Contabilidade e Atuaria da \\ Faculdade de Economia e Administração e Contabilidade da USP
}

\section{Introdução:}

O modelo econômico tradicional utilizado pelas empresas objetivas principalmente determinar o nível ótimo de produção de uma empresa, que fabrica um único produto ainda, o melhor "mix" de produção de uma empresa com multiprodutos.

Este modelo econômico tradicional considera que todas as variáveis de entrada, ou sejam: preços de venda, custos e volumes de produção são conhecidos com certeza. Não inclui portanto, um ajustamento para os fatores risco e incerteza limitada, dessa forma, sua utilidade uma vez que o mundo é repleto de incertezas.

No Brasil, grande parte das empresas, utiliza-se de modelo para efeitos gerenciais, entretanto, são raros os casos em que o empregado um modelo formal que leve em conta o fator incerteza, embora este esteja um país em que este fator é predominante.

O Objetivo primordial deste trabalho é apresentar um modelo econômico em condições de incerteza quanto ao volume de vendas dos produtos da empresa. Considera-se o caso de uma surpresa com multiprodutos em que há interdependência entre as demandas desses produtos. Través da aplicação desse modelo pode-se saber qual a maior probabilidade de alcançar determinada faixa de lucro.

O modelo econômico da empresa é dado por:

$$
\begin{aligned}
& L=(P-V) \cdot X-F \quad \text { (1) ou } \\
& L=C \cdot X \quad-F
\end{aligned}
$$

A análise tradicional supõe que todos os dados que o modelo acima requer são conhecidos exatamente. Mas na vida real isto é improvável e cada um dos fatores mencionados pode se constituir numa variável aleatória.

A palavra aleatória é usada para indicar que o valor que tal variável assume num experimento depende da ocorrência do experimento que, por sua vez, depende do acaso (Hoel, 1963).

O fato de o volume de vendas se comportar como uma variável aleatória significa que as previsões relativas as unidades a serem vendidas para cada produto não se constituem num único dado, mas se distribuem dentro de uma determinada faixa. 
Como exemplo, suponha que uma surpresa fabrique os produtos I e II.

Considere que os volumes de vendas previstos para cada um destes produtos podem ser qualquer um dos valores apresentados na $1^{\circ}$ coluna no quadro 1.

\section{Quadro 1 Volumes Esperados de Vendas dos Produtos 1 e 2}

\begin{tabular}{|l|l|l|l|l|}
\hline $\begin{array}{l}\text { Volume } \\
\text { vendas } \\
\text { (Unidades) } \\
(1)\end{array}$ & $\begin{array}{l}\text { Distribuição de } \\
\text { probabilidades } \\
\text { Produto I } \\
(2)\end{array}$ & $\begin{array}{l}\text { Volume esperado } \\
\text { de vendas } \\
\text { Produto1 } \\
(3)=(1) \times(2)\end{array}$ & $\begin{array}{l}\text { Distribuição de } \\
\text { Probabilidades } \\
\text { Produto II }\end{array}$ & $\begin{array}{l}\text { Volume Esperado } \\
\text { de Vendas } \\
\text { Produto II } \\
(5)=(1) \times(4)\end{array}$ \\
\hline 10.000 & 0,1 & 1.000 & 0,3 & 3.000 \\
15.000 & 0,2 & 3.000 & 0,4 & 6.000 \\
20.000 & 0,3 & 6.000 & 0,2 & 4.000 \\
25.000 & 0,2 & 5.000 & 0,1 & 2.500 \\
30.000 & 0,1 & 3.000 & -- & - \\
35.000 & 0,1 & 3.000 & -- & -- \\
& ------ & - \\
& 0,1 & -21.500 & 1,0 & 15.500 \\
\hline
\end{tabular}

Como se vê no Quadro 1, para cada volume pode-se associar uma probabilidade de ocorrência conforme se considere o produto I (coluna 1) ou o produto II (coluna 4).

As distribuições de probabilidades podem ser obtidas de diversas formas. Uma delas, por exemplo, é a utilização de dados históricos de produtos similares existentes no mercado.

Quando não existirem produtos similares pode-se atribuir probabilidades de forma Subjetiva a cada um dos produtos. É possível também designar um grupo de especialistas internos ou externos para á empresa para tal atribuição.

Os volumes esperados de vendas são calculados multiplicando-se os volumes de vendas em unidades pelas respectivas probabilidades e somando-se os produtos obtidos. Os resultados encontra-se na coluna 03 (Produto I) e coluna 5 (Produto II) ao Quadro 1.

Se fosse feita a escolha entre os produtos entre os produtos com base apenas nessas informações , 0 administrador optaria pelo produto I, que é o que apresenta o maior volume esperado de vendas , ou seja 21.500 unidades contra 15.500 unidades do produto II.

Suponha agora um exemplo, em que o volume esperado de venda dos dois produtos é idêntico, ou seja, 2.000 unidades, conforme Quadro 2.

Quadro 2 Volume Esperado de Vendas dos Produtos I e II

\begin{tabular}{|c|c|c|c|c|}
\hline $\begin{array}{ll}\text { Volume } & \text { de } \\
\text { Vendas } & \\
\text { (Unidades) } & \\
\text { (1) } & \end{array}$ & $\begin{array}{l}\text { Distribuição de } \\
\text { Probabilidades } \\
\text { Produto I } \\
\text { (2) }\end{array}$ & $\begin{array}{l}\text { Volume Esperado } \\
\text { de Vendas } \\
\text { Produto } 1 \\
(3)=(1) \times(2)\end{array}$ & $\begin{array}{l}\text { Distribuição de } \\
\text { Probabilidades } \\
\text { Produto II } \\
(4) \\
\end{array}$ & $\begin{array}{l}\text { Volume Esperado } \\
\text { de Vendas } \\
\text { Produto II } \\
(5)=(1) \times(4)\end{array}$ \\
\hline $\begin{array}{l}1.000 \\
1.500 \\
2.000 \\
2.500 \\
3.000\end{array}$ & $\begin{array}{l}0,1 \\
0,2 \\
0,4 \\
0,2 \\
0,1 \\
-- \\
1,0\end{array}$ & $\begin{array}{l}100 \\
300 \\
800 \\
500 \\
300 \\
--- \\
2.000\end{array}$ & $\begin{array}{l}- \\
-- \\
1,0 \\
-- \\
-- \\
-- \\
1,0\end{array}$ & $\begin{array}{l}- \\
-- \\
2.000 \\
-- \\
-- \\
---- \\
2.000\end{array}$ \\
\hline
\end{tabular}


Embora o volume esperado de vendas seja o mesmo para os dois produtos, ou seja $\$ 2.000$, o administrador não ficaria indiferente na escolha entre eles. Suponha neste caso que o preço de venda por unidade (P) seja de $\$ 1.000$, o custo variável (V) por unidade seja de $\$ 600$ e o custo fixo total (F) $\$ 500.000$, para ambos os produtos.

Substituindo-se esses dados no modelo econômico (1) e analisando-se, por exemplo, que no caso do produto I há 0,1 (ou $10 \%$ ) de probabilidade de haver prejuízo de $\$ 100.000$ (isto é, $(\$ 1.000-\$ 1.000) \times 1.000$ um. - \$500.000).

Enquanto isso, há $100 \%$ de probabilidade de que os lucros do produto II se situem em $\$ 300.000$ (ou seja, $(\$ 1.000$ - \$600) x 2.000 um - \$500.000).

Há também o produto I, 0,3 (30\%) de probabilidade de que as vendas sejam maiores que 2.000 unidades. Nesse caso os lucros do produto I serão maiores que os do produto II.

Conclui-se que, além da informação quanto ao volume esperado de vendas, é importante quanto ao volume esperado de vendas, é importante conhecer o grau de dispersão da distribuição de probabilidades. Quanto maior for o grau de dispersão maior será o risco envolvido.

A medida quantitativa do grau de dispersão da distribuição de probabilidade é dada pela variância, simbolizada por 02. Pode-se usar também o desvio padrão (o) que é a raiz quadrada da variância.

Geralmente, a demanda ou volume de vendas, é o fator que apresenta o maior grau de incerteza quanto a sua realização. Por esse motivo, e também para que se possa fazer uma análise simplificada, assume-se que esses sejam o único fator, entre os mencionados, que se comporta como uma variável aleatória. Portanto, neste trabalho, os demais fatores serão considerados como constantes, claro que isso pode ser valido somente para um intervalo relevante de produção.

Neste trabalho será analisado um modelo para a empresa que vende "n" produtos no mercado. Supõe-se que há correlação entre as demandas ou volumes esperados de vendas desses produtos.

\section{Caso de Correção Positiva entre as Demandas dos Produtos}

Uma empresa que atualmente fabrica o produto a estuda a alternativa de introduzir os produtos B e C no mercado.

A seguir no quadro 3, apresentam-se os dados necessários para a analise,

Quadro 3 - Dados para a Análise de Introdução de Novos Produtos

\begin{tabular}{|c|c|c|c|c|c|c|}
\hline produtos & $\begin{array}{l}\text { Vol.Esper. } \\
\text { De } \\
\text { Vendas } \\
\text { (u) }\end{array}$ & $\begin{array}{l}\text { Variância do } \\
\text { Volume } \\
\text { Esperado de } \\
\text { Vendas } \\
\text { (o2) }\end{array}$ & $\begin{array}{l}\text { Preço de } \\
\text { Venda p/ } \\
\text { Unidade } \\
(\$) \\
(\mathrm{V})\end{array}$ & $\begin{array}{l}\text { Custo } \\
\text { Variável p/ } \\
\text { unidade } \\
\text { (\$) } \\
\text { (V) }\end{array}$ & $\begin{array}{ll}\text { Margem } & \text { de } \\
\text { Contr. } & \mathrm{P} / \\
\text { Unidade } & \\
\text { (\$) } & \\
\text { (C) } & \end{array}$ & $\begin{array}{l}\text { Custo } \\
\text { Fixo } \\
\text { Total } \\
(\$) \\
(\mathrm{F}) \\
\end{array}$ \\
\hline $\begin{array}{l}A \\
B \\
C\end{array}$ & $\begin{array}{l}4.800 \\
4.800 \\
4.800\end{array}$ & $\begin{array}{l}(452) 2 \\
(350) 2 \\
(250) 2\end{array}$ & $\begin{array}{l}1.800 \\
1.800 \\
1.800\end{array}$ & $\begin{array}{l}1.000 \\
1.000 \\
1.000\end{array}$ & $\begin{array}{l}800 \\
800 \\
800\end{array}$ & $\begin{array}{l}3.500 .000 \\
3.500 .000 \\
3.500 .000\end{array}$ \\
\hline
\end{tabular}

Analisando-se dados do Quadro3, verifica-se que os três produtos apresentam o mesmo volume de vendas esperado. Os preços unitários e os custos variáveis por unidade também são iguais para os três produtos gerando, em conseqüência, a mesma margem de contribuição.

Considera-se por simplificação, que o custo fixo total não se altera com a introdução dos novos produtos.

Assim, o único dado que apresenta diferença em relação a cada produto é a variância dos volumes esperados de venda. Isso significa que os volumes esperados de venda são idênticos mas o grau de dispersão dos dados em relação a média varia de produto para produto. 
Adição dos Produtos B ou C

No caso da adição do produto $B$ ou $C$ ao produto já existente, o lucro total esperado $E$ (L) é dado pela expressão a seguir, adaptada a partir da expressão (2):

$E[L]=C i U i+C j U j-F i-F j$

Onde:

$\mathrm{I}=\mathrm{A}$ e $\mathrm{J}=\mathrm{B}$ no caso da adição do produto $\mathrm{B}$ ou então

$\mathrm{I}=\mathrm{A}$ e $\mathrm{J}=\mathrm{C}$ para o caso da adição do produto $\mathrm{C}, \mathrm{e}$

$\mathrm{U} \mathrm{i}, \mathrm{j}$ = volume esperado do produto $\mathrm{i}$ ou $\mathrm{j}$

Considerando a adição do produto $B$ por exemplo, o lucro esperado é assim calculado:

$E[L]=(\$ 800)(4.800$ un $)+(\$ 800)(4.800$ un $)-\$ 3.500 .000-3.500 .000$

$E[L]=\$ 680.000$

Por outro lado, a variância do lucro Var [L] é dada por (JOHNSON e SIMIK, 1971):

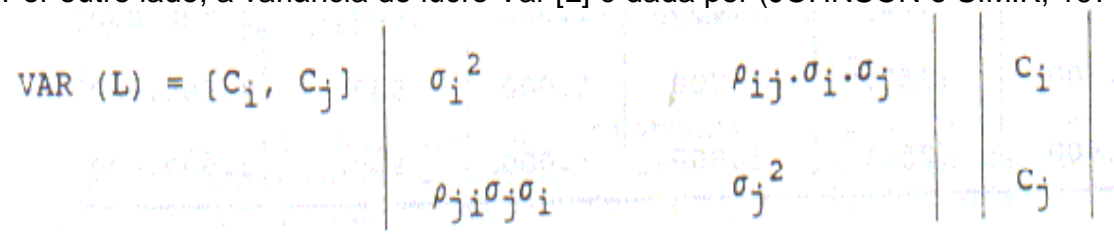

Onde: pij mede o grau de correlação entre os volumes de venda dos produtos A e B.

Efetuando-se a multiplicação dos elementos acima, vem:

$$
\sigma^{2}(L)=C_{i}{ }^{2} \cdot \sigma_{i}{ }^{2}+2 \rho_{i j} \sigma_{i} \sigma_{j} C_{i} C_{j}+C_{j}{ }^{2} \sigma_{j}{ }^{2}
$$

Ou então : para ocaso de obtenção do desvio padrão do lucro o $(L)$ :

$$
\rho_{12}=0,50, \rho_{13}=0,50 . \rho_{23}=0,50
$$

A titulo de exemplo será efetuada uma aplicação prática em que se calculará o desvio padrão do lucro para o caso da introdução do produto B.

Substituindo os elementos de expressão (5) pelos respectivos valores, vem:

$$
\sigma(L)=\left((800)^{2}(450)^{2}+(2)(0,50)(450)(350)(800)(800)+(800)^{2} \cdot(350)^{2}\right)^{1 / 2}=\$ 555.698
$$

Adição simultânea dos Produtos A e B

Para o caso da adição simultânea dos produtos $\mathrm{B}$ e $\mathrm{C}$ ao produto $\mathrm{A}$ já existente, tem-se:

Para o lucro esperado, E (L):

$$
E(L)=C_{1} H_{1}+C_{2} H_{2}+C_{3} H_{3}-F_{1}-F_{2}-F_{3}
$$

Onde:

$1=$ Produto $A$

$2=$ Produto $B$

$3=$ Produto $\mathrm{C}$

Para a variância do lucro,o2, (L) (JOHNSON e SIMIK, 1971) 


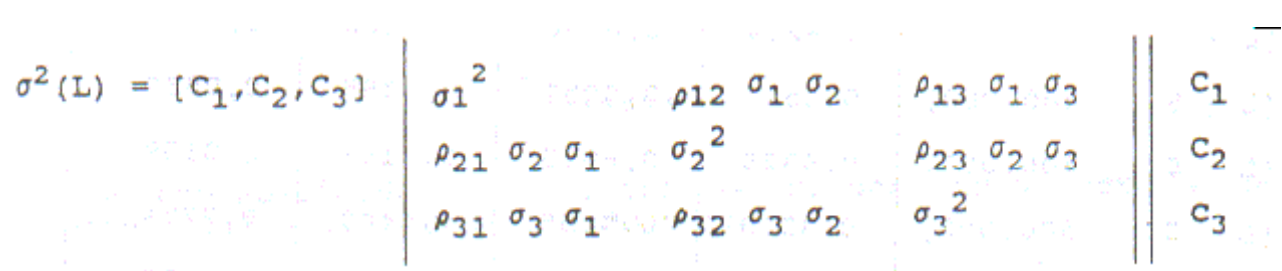

Efetuando-se a multiplicação dos elementos acima e considerando o desvio padrão do lucro o (L) _

$$
\sigma(L)=\left(c_{1}{ }^{2} \cdot \sigma_{1}{ }^{2}+c_{2}{ }^{2} \sigma_{2}{ }^{2}+c_{3}{ }^{2} \sigma_{3}{ }^{2}+2 \rho_{12} c_{1} \sigma_{1} c_{2} \sigma_{2}+2 \rho_{13} c_{1} \sigma_{1} \cdot c_{3} \sigma_{3}+2 \rho_{23} c_{2} \sigma_{2} c_{3} \cdot \sigma_{3}\right)^{1 / 2}
$$

Substituindo-se os elementos das expressões acima pelos respectivos valores, são determinadas as probabilidades de obtenção de vários níveis de lucro e de vários níveis de prejuízo. Os resultados estão sumarizados no quadro 4.

Quadro 4

Análise das Probabilidades de Adição de Novos Produtos correlação Positiva entre as Demandas dos Produtos.

\begin{tabular}{|c|c|c|c|c|}
\hline $\begin{array}{r}\text { PROD. } \\
\text { A }\end{array}$ & $\begin{array}{l}\text { ADIÇÃO } \\
\text { DO } \\
\text { PROD } \\
\text { B }\end{array}$ & $\begin{array}{l}\text { ADIÇÃO } \\
\text { DO } \\
\text { PROD. } \\
\text { C }\end{array}$ & $\begin{array}{l}\text { ADIÇÃO } \\
\text { DOS } \\
\text { PRODUTOS } \\
\text { B E C }\end{array}$ & \\
\hline $\begin{array}{l}\text { Lucro Esperado - E (L) } \\
\text { Desvio Padrão do Lucro - oL }\end{array}$ & $\begin{array}{l}\$ 340.000 \\
\$ 360.000\end{array}$ & $\begin{array}{l}\$ 680.000 \\
\$ 555.698\end{array}$ & $\begin{array}{l}\$ 680.000 \\
\$ 491.528\end{array}$ & $\begin{array}{l}\$ 1.020 .000 \\
\$ 690.507\end{array}$ \\
\hline $\begin{array}{l}\text { Probabilidades: } \\
\text { p }\{L>>0\} \\
\text { p }\{L>\$ 200.000\} \\
\text { p }\{L>\$ 400.000\} \\
\text { p }\{L>\$ 500.000\} \\
\text { p }\{L>\$ 1.000 .000\} \\
\text { p }\{L>\$ 1.300 .000\} \\
\text { p }\{L>\$ \quad 0\} \\
\text { p }\{L>\$ 150.000\} \\
\text { p }\{L>\$ 300.000\}\end{array}$ & $\begin{array}{l}0,8888 \\
0,6517 \\
0,4325 \\
0,3300 \\
0,0336 \\
0,0038 \\
0,1736 \\
0,0869 \\
0,0375\end{array}$ & $\begin{array}{l}0,8888 \\
0,8051 \\
0,6915 \\
0,6255 \\
0,2810 \\
0,1314 \\
0,1112 \\
0,0681 \\
0,0392\end{array}$ & $\begin{array}{l}0,9162 \\
0,8365 \\
0,7157 \\
0,6443 \\
0,2578 \\
0,1038 \\
0,0838 \\
0,0455 \\
0,0233\end{array}$ & $\begin{array}{l}0,9306 \\
0,8830 \\
0,8159 \\
0,7734 \\
0,5120 \\
0,3409 \\
0,0694 \\
0,0455 \\
0,0281\end{array}$ \\
\hline
\end{tabular}

Para a elaboração do Quadro 4 são utilizadas as tabelas de distribuição normal depois de converter as variáveis normais em sua forma reduzida. Para tanto se utilizando da seguinte equação para o cálculo de $Z$.

$$
Z=\frac{L-E(L) N(0,1)}{O(L)} N(0,1)
$$

A Titulo de exemplo, considere o cálculo da probabilidade de os lucros serem maiores ou iguais a zero para o produto ${ }^{a}$ Essa probabilidade pode ser calculada considerando-se a área de distribuição à direita do lucro zero, conforme Figura 1. 


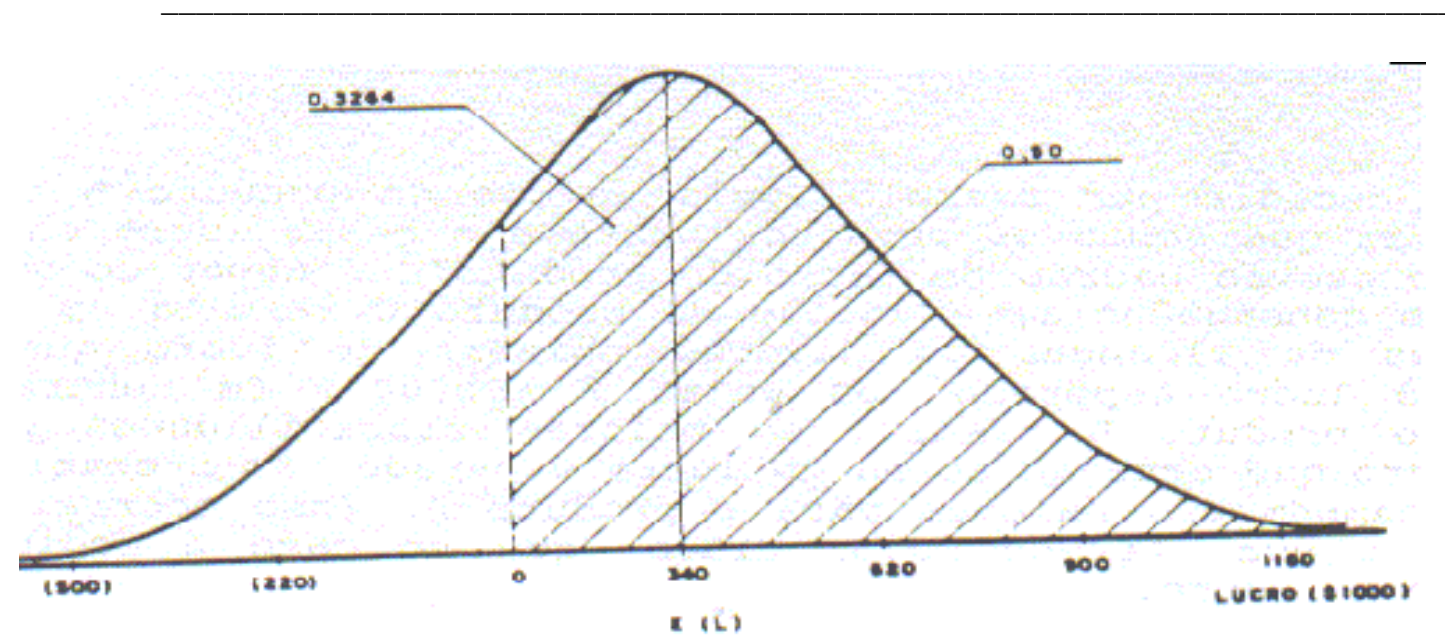

A probabilidade de o lucro se situar entre o zero e o valor esperado

$(E[L]=\$ 340.000)$

é dado por:

$z=\frac{0-\$ 340.000}{\$ 360.000}=-0,94$

Os $\$ 360.000$ correspondem ao valor do desvio padrão do lucro (oL) para o produto A.

Consultando o valor de z numa tabela de distribuição normal, que se encontra em qualquer livro de estatística, encontra-se 0,3264 , isto é, a probabilidade de o lucro se situar entre zero e o valor esperado são de $32,64 \%$.

Para achar a probabilidade de o lucro ser maior que zero, basta adicionar ao valor já encontrado $(0,3264)$, o valor correspondente à área total situada à direita do lucro esperado, ou seja, 0,50. Esse valor corresponde a metade da área da curva normal cujo total é igual a unidade. Como a curva normal é simétrica, metade desta área $(0.50)$ se situa à direita e metade $(0,50)$ se situa à esquerda do valor esperado. Este valor encontra-se exatamente no meio da curva e corresponde à média da distribuição.

Assim a probabilidade de se obter um lucro maior ou igual a zero é de $0,3364+0,50=0,8264 \%$ (vide figura 1).

A segunda coluna do Quadro 4 mostra as probabilidades de lucro do produto A já existente. A terceira e quarta colunas apresentam as probabilidades de lucro adicionando-se o produto $B$ (Coluna 3 ) ou o produto $O$ (coluna 4), supondo a existência do produto A. A quinta coluna mostra as probabilidades de lucros pela adição simultânea dos produtos 3 e C.

Para se decidir por exemplo, pela adição do produto 3 ou O, o Quadro 4 mostra que ambos os produtos possuem o mesmo lucro esperado. Entretanto, o desvio padrão do lucro do produto C é menor do que o do produto 3. Em conseqüência, a adição do produto $C$ resulta em maiores probabilidades de alcançar os diversos níveis de lucro que sejam inferiores ao lucro esperado, ou seja, $\$ 680.000$. Em contraste, a introdução do produto B resulta em maiores probabilidades para os níveis de lucro que estejam acima do lucro esperado. As probabilidades de prejuízo também são maiores para o produto B. Isso ocorre porque, esse produto é que oferece maior risco, que no caso é medido pelo desvio padrão do lucro.

A última coluna do Quadro 4 considera a adição simultânea de ambos os produtos. Como se verifica, o desvio padrão do lucro é maior que nas alternativas anteriores. Mas isso não implica em menores probabilidades de alcançar os diversos níveis de lucro. Isso se deve, apenas, ao fato de que o lucro esperado também cresceu pela adição dos dois produtos. Na verdade, o que ocorreu foi o aumento das probabilidades

Caderno de Estudos no05, São Paulo, FIPECAFI - Junho/1992 
de alcançar os diversos níveis de lucro. Além disso, de maneira geral, houve uma redução das probabilidades de prejuízos. Esse resultado é consistente com a teoria dos portfólios e salienta a necessidade de se considerar a inter-relação entre as demandas dos produtos na análise. 11

2. Assumindo Correlação Negativa entre as Demandas dos Produtos

Para evidenciar os efeitos da existência de correlação negativa e positiva entre produtos, serão considerados os mesmos dados do caso anterior, exceto que a área de "marketing especificou que as correlações de demanda entre os produtos são as seguintes":

$\mathrm{p} 12=-0,50,413=-0.50$, e p23 0,50 . Agora os produtos B e C são correlacionados negativamente com o produto A (existente). Entretanto são correlacionados positivamente uni com o outro. As probabilidades de adicionar ou outros produtos são apresentados no Quadro 5.

Análise das Probabilidades de Adição de Novos Produtos Correlação Negativa Entre os Produtos

quadro 5

\begin{tabular}{|c|c|c|c|c|c|}
\hline & $\begin{array}{c}\text { ADIÇÃO } \\
\text { DO } \\
\text { PRODUTO } \\
\text { A }\end{array}$ & $\begin{array}{r}\text { ADIC } \\
\mathrm{C} \\
\mathrm{PRO} \\
\mathrm{B}\end{array}$ & $\begin{array}{l}\text { ÃO } \\
\text { UTO }\end{array}$ & $\begin{array}{c}\text { ADIÇÃO } \\
\text { DO } \\
\text { PRODUTO } \\
\text { C }\end{array}$ & $\begin{array}{l}\text { ADIÇÃO } \\
\text { DOS } \\
\text { PRODUTOS } \\
\text { B E C }\end{array}$ \\
\hline $\begin{array}{l}\text { Lucro Esperado - E(L) } \\
\text { Desvio Padrão do Lucro- oL }\end{array}$ & $\begin{array}{l}\$ 340.000 \\
\$ 360.000 \\
\end{array}$ & $\begin{array}{l}\$ 680.000 \\
\$ 327.41 \\
\end{array}$ & $\begin{array}{l}\$ 68 \\
\$ 31\end{array}$ & .000 & $\begin{array}{c}\$ 1.020 .000 \\
\$ 362.215 \\
\end{array}$ \\
\hline 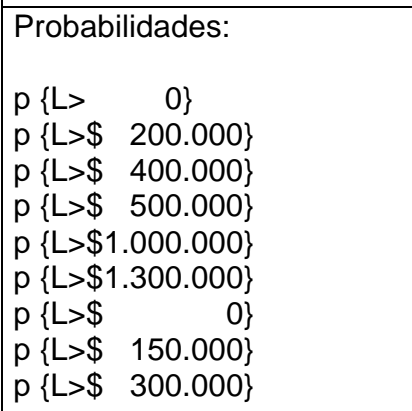 & $\begin{array}{l}0,8264 \\
0,6517 \\
0,4325 \\
0,3300 \\
0,0336 \\
0,0038 \\
0,1736 \\
0,0869 \\
0,0375\end{array}$ & $\begin{array}{l}0,9812 \\
0,9292 \\
0,8051 \\
0,7088 \\
0,1635 \\
0,0294 \\
0,0188 \\
0,0055 \\
0,0014\end{array}$ & $\begin{array}{l}0,98 \\
0,93 \xi \\
0,815 \\
0,715 \\
0,135 \\
0,02 \\
0,01 \\
0,00 \\
0,00\end{array}$ & & $\begin{array}{l}0,9976 \\
0,9881 \\
0,9564 \\
0,9251 \\
0,5239 \\
0,2206 \\
0,0024 \\
0,0006 \\
0,0001\end{array}$ \\
\hline
\end{tabular}

Comparando-se a adição do produto C com a do B no Quadro 5, verifica-se que os menores desvios padrão de lucro do produto $\mathrm{C}$ proporcionam probabilidades um pouco maiores de alcançar os diversos níveis de lucro que se situam abaixo do lucro esperado, e também menores probabilidades de ocorrência de prejuízos.

A última coluna mostra que a adição de ambos os produtos resulta em altas probabilidades de atingir os diversos níveis de lucro, que se situam abaixo do lucro esperado.

Além disso, as probabilidades de ocorrência de prejuízos se aproximam de zero.

$\mathrm{Na}$ verdade, esse fato é consistente com a teoria portfólios uma vez que a correlação negativa da demanda entre o produto $\mathrm{A}$ e os produtos a serem adicionados causam menores desvios padrão de lucros. 
Os demonstrativos apresentados proporcionam à administração as informações de probabilidades que podem auxiliar na tomada de decisões. Mas a escolha de uma das alternativas depende do grau de aversão ao risco por parte do administrador.

\section{CONCLUSÕES}

O modelo econômico tradicional considera que suas variáveis componentes são conhecidas com certeza, ignorando dessa forma os fatores risco e incerteza.

Porém, para a construção de modelos mais adaptáveis à realidade é necessário considerar que as variáveis componentes podem assumir um comportamento aleatório.

Os modelos determinísticos continuam a apresentar grande utilidade. Entretanto, os modelos probabilísticos, por serem mais ajustados à realidade, dotam a administração de instrumentos mais úteis ao processo de tomada de decisões porque permitem conhecer por exemplo, qual o produto que apresenta a menor probabilidade de atingir prejuízo, ou a maior probabilidade de alcançar determinada faixa de lucro.

Um problema inicial que se apresenta para o desenvolvimento de modelos probabilísticos é que, estes requerem o conhecimento da. forma de distribuição de probabilidades não só das variáveis de entrada, ou sejam: preço unitário de venda, custo unitário variável, volume de produção ou de vendas e custo fixo total, como também da variável resultante, ou seja, o lucro total.

No desenvolvimento deste trabalho adotou-se a hipótese simplificadora da distribuição normal e foi considerado o caso também simplista de que a demanda do produto é a única variável aleatória do modelo. Analisou-se a situação de interdependência entre as demandas dos produtos para uma empresa com multiprodutos. A forma utilizada para medir o risco foi o desvio padrão e a variância do lucro.

Assim foram obtidas as probabilidades de se atingir diversas faixas de lucro e de prejuízo nos casos de correlação positiva e negativa entre os produtos.

Constatou-se que o instrumento analisado pode ser bastante útil no processo decisório das empresas.

\section{BIBLIOGRAFIA}

CORRAR, Luiz João. "Análise das Relações Custo-Volume-Lucro para Multiprodutos em Condições de". Incerteza". Tese de Doutoramento Universidade de São Paulo, Faculdade de Economia, Administração e Contabilidade, 1990.

HOEL, P.G., "Estatística Elementar", Rio de Janeiro, Editor Fundo de Cultura, 1963.

JAEDICKE, E.K. e Robichek, A.A. "Cost-Volunie-Profit Analysis under Conditions of Uncertainty." The Accountiflg Review, (October, 1964)

JOHNSON, G.L., e SIMIK, S.S. "Multiproduct CVP Analysis under Uncertainty". Decision Sience. July, 1973. 